\title{
Optimal power allocation for downlink two-user non-orthogonal multiple access in visible light communication
}

\author{
Hong Shen*, Yanfei Wu, Wei Xu, Chunming Zhao \\ National Mobile Communications Research Laboratory, Southeast University, Nanjing 210096, China \\ * Corresponding author, Email: shhseu@seu.edu.cn
}

\begin{abstract}
In this work, we consider employing the NOMA (Non-Orthogonal Multiple Access) technique in downlink VLC (Visible Light Communication) for performance enhancement. In particular, focusing on a typical NOMA scenario with two users, we optimize the power allocation strategies under both sum-rate maximization and max-min fairness criteria, where practical optical power and QoS (Quality of Service) constraints are included. As our main contribution, we achieve optimal power allocation solutions in semi-closed forms via mathematical analysis, which, to the best of our knowledge, have not been reported in literature. Simulation results demonstrate that NOMA can provide remarkable performance gains over OMA (Orthogonal Multiple Access) in the context of VLC downlinks.
\end{abstract}

Keywords: NOMA, VLC, power allocation, sum-rate maximization, max-min fairness

\section{Introduction}

VLC, which is a potential candidate for future wireless communications, has drawn increasing attention from both academia and industry. Compared to traditional RF (Radio Frequency) communications, VLC enjoys multiple advantages such as unlicensed spectrum, high secrecy, and immunity to $\mathrm{RF}$ interference ${ }^{[1-3]}$. On the other hand, the NOMA scheme has been recognized as a powerful technique for improving the performance of conventional OMA based communication systems. The main feature of NOMA is that all users' signals are multiplexed in the power domain at the transmitter, and they are detected by SIC (Successive Interference Cancellation) at the receiver ${ }^{[4-6]}$.

With respect to NOMA in RF wireless communications, a number of studies have focused on devising power allocation strategies to enhance the system performance $^{[7-9]}$. The max-min fairness based power allocation was studied in Ref. [7] under both instantaneous and average CSI (Channel State Information). Alternatively, the authors in Ref. [8] considered maximizing the sum rate of the NOMA system with two users. Moreover, a general power allocation was proposed in Ref. [9] for two-user NOMA by

Manuscript received May 20, 2017; accepted Jul. 04, 2017

This work is supported by the National Basic Research Program of China (973 Program) (No. 2013CB329204), the National Natural Science Foundation of China (Nos. 61501110, 61471114, 61461136003, 61571118, and 61521061), the Natural Science Foundation of Jiangsu Province (No. BK20150635), and the Fundamental Research Funds for Central Universities (No. 2242016K41080). 
ensuring that each user has a larger rate than that of OMA.

The power allocation optimization for VLC NOMA systems has also been investigated in some recent works such as Refs. [10-12]. The authors in Ref. [10] proposed an efficient GRPA (Gain Ratio Power Allocation) strategy for VLC NOMA. Then, for NOMA in VLC, the sum logarithmic user rate maximized power allocation was developed in Ref. [11] using the Lagrangian dual method. In Ref. [12], the authors applied the gradient projection algorithm to address the QoS constrained power allocation problems, which maximize the sum rate and minimum user rate.

In this study, we optimize the power allocation for downlink VLC NOMA systems with two users. By incorporating both optical power and QoS constraints, we respectively maximize the sum rate and minimum user rate, which are both important performance measures. First, we analyze the feasibility condition for the considered optimization problems. Furthermore, we derive their optimal solutions in semi-closed forms that can be efficiently computed, and this has not been reported in existing literature. Compared to the traditional OMA transmission, the proposed NOMA schemes can provide a higher sum rate or fairness rate, as validated via simulations.

\section{System model description}

We study a downlink VLC system with one LED (Light-Emitting Diode) and two users, as depicted in Fig. 1. The LED communicates with the two users using the NOMA technique, which has been shown to outperform the conventional OMA scheme ${ }^{[4-6]}$. A detailed description of the considered system model is provided below.

The LED transmitter first superimposes the transmit signals of the two users by $x=\sqrt{p_{1}} s_{1}+\sqrt{p_{2}} s_{2}$, where $s_{k} \in[-1,1], k=1,2$ denotes the transmit symbol of the $k$ th user and $p_{k} \geqslant 0$ represents the corresponding transmit power. Then, a DC (Direct Current) bias $P_{o}$ is added to $x$ to generate the LED input such that it is nonnegative.

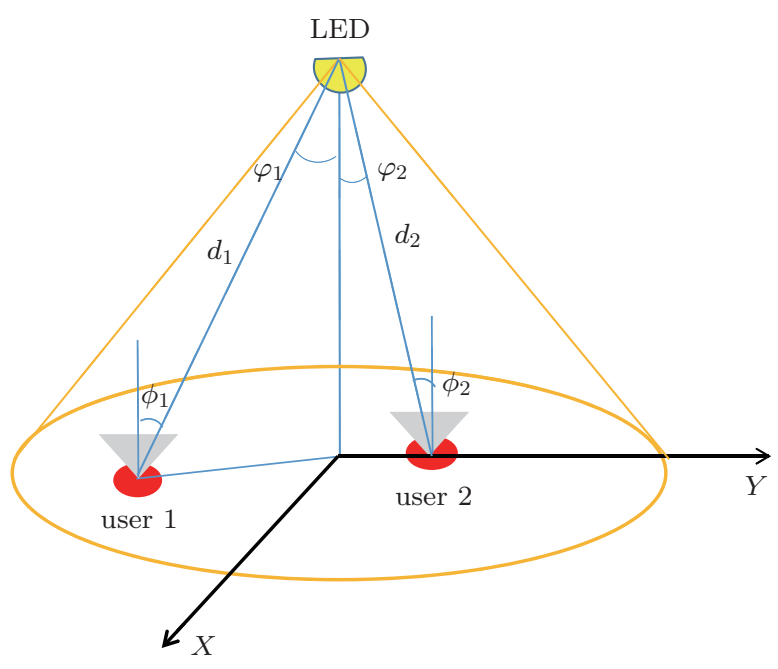

Figure 1 System model

For VLC channels, it has been shown that the power of the NLOS (Non-Line-of-Sight) signals is much weaker than that of the LOS signal ${ }^{[13]}$. Thus, we only need to focus on the LOS channel gain. Specifically, the channel between the LED and the $k$ th user is modeled by ${ }^{[13,14]}$

$$
h_{k}=\frac{(m+1) A R_{P}}{2 \pi d_{k}^{2}} \cos ^{m}\left(\psi_{k}\right) T\left(\phi_{k}\right) g\left(\phi_{k}\right) \cos \left(\phi_{k}\right),
$$

where $m$ is the Lambertian emission order of LED, $A$ represents the detection area of the PD (Photodiode), $R_{P}$ denotes the responsivity of the $\mathrm{PD}, d_{k}$ is the distance between the LED and user $k, \psi_{k}$ and $\phi_{k}$ represent the LED irradiance angle and the PD incidence angle respectively, $T\left(\phi_{k}\right)$ is the gain of the optical filter, and $g\left(\phi_{k}\right)$ represents the gain of the optical concentrator, which is given by

$$
g\left(\phi_{k}\right)= \begin{cases}\frac{n^{2}}{\sin ^{2}\left(\Psi_{\mathrm{FOV}}\right)}, & 0 \leqslant \phi_{k} \leqslant \Psi_{\mathrm{FOV}}, \\ 0, & \phi_{k}>\Psi_{\mathrm{FOV}},\end{cases}
$$

where $n$ is the reflective index of the optical concentrator and $\Psi_{\mathrm{FOV}}$ is the receiver FOV (Field-of-View). Because VLC channels are known to be slow varying and the corresponding estimation error can be relatively small, we assume that perfect CSI is available at the transmitter, as reported in Refs. [10-12].

After removing the $\mathrm{DC}$ components, the received 
signals of the two users are

$$
\begin{aligned}
& y_{1}=\sqrt{p_{1}} h_{1} s_{1}+\sqrt{p_{2}} h_{1} s_{2}+z_{1}, \\
& y_{2}=\sqrt{p_{2}} h_{2} s_{2}+\sqrt{p_{1}} h_{2} s_{1}+z_{2},
\end{aligned}
$$

where $z_{k}, k=1,2$ is the noise term, which is a realvalued zero-mean Gaussian variable, with its variance defined by

$$
\begin{aligned}
\sigma_{z_{k}}^{2}= & 2 q\left(h_{k} P_{o}+2 \pi A R_{P} \chi_{\mathrm{amb}} g\left(\phi_{k}\right)\right. \\
& \left.\times\left(1-\cos \left(\Psi_{\mathrm{FOV}}\right)\right)\right) B+i_{\mathrm{amp}}^{2} B,
\end{aligned}
$$

where $q$ is the electronic charge, $\chi_{\mathrm{amb}}$ is the ambient light photocurrent, $i_{\mathrm{amp}}$ is the pre-amplifier noise current density, and $B$ is the system bandwidth. Without loss of generality, we assume that $h_{1} / \sigma_{z_{1}} \leqslant h_{2} / \sigma_{z_{2}}$, i.e., user 2 is stronger. Then, user 2 should carry out SIC to remove the interference from user 1, while user 1 does not need to perform SIC. Accordingly, Eq. (4) becomes

$$
y_{2}=\sqrt{p_{2}} h_{2} s_{2}+z_{2}
$$

Based on Eq. (3), Eq. (6), and Eq. (8) in Ref. [15], we obtain the achievable rates of the two users as

$$
\begin{gathered}
R_{1}\left(p_{1}, p_{2}\right)=\frac{1}{2} \mathrm{lb}\left(1+\frac{2 h_{1}^{2} p_{1}}{\pi e\left(h_{1}^{2} p_{2} / 3+\sigma_{z_{1}}^{2}\right)}\right), \\
R_{2}\left(p_{2}\right)=\frac{1}{2} \mathrm{lb}\left(1+\frac{2 h_{2}^{2} p_{2}}{\pi e \sigma_{z_{2}}^{2}}\right) .
\end{gathered}
$$

It should be noted that the above rate expressions are derived by taking into account the finite amplitude of the transmit symbols (see Ref. [15]). In the next section, we aim to find the optimal power allocation under two different design criteria, namely, sum-rate maximization and max-min fairness.

\section{Optimal power allocation strategies for VLC NOMA}

\subsection{Sum-rate maximized power alloca- tion}

The sum-rate maximization problem is first studied for the VLC NOMA system described in section 2 .
Because the transmitted signal should be nonnegative in VLC systems, we have $x^{\prime}=\sqrt{p_{1}} s_{1}+\sqrt{p_{2}} s_{2}+$ $P_{o} \geqslant 0$, where the DC component $P_{o}$ is used for illumination and the AC (Alternating Current) component $\sqrt{p_{1}} s_{1}+\sqrt{p_{2}} s_{2}$ is used for communication. Recall that $s_{1}, s_{2} \in[-1,1]$. Then, it can be inferred that $\sqrt{p_{1}}+\sqrt{p_{2}} \leqslant P_{o}$ holds such that $x^{\prime} \geqslant 0$. Moreover, we also impose QoS constraints on both users' achievable rates. Accordingly, the optimization problem can be formulated as

$$
\begin{array}{cc}
\max _{p_{1}, p_{2}} & R_{1}\left(p_{1}, p_{2}\right)+R_{2}\left(p_{2}\right), \\
\text { s.t. } & \\
& \sqrt{p_{1}}+\sqrt{p_{2}} \leqslant P_{o}, \\
& R_{1}\left(p_{1}, p_{2}\right) \geqslant \tilde{R}_{1}, \\
& R_{2}\left(p_{2}\right) \geqslant \tilde{R}_{2},
\end{array}
$$

where $\tilde{R}_{1}$ and $\tilde{R}_{2}$ denote the minimum required rates for both users. Problem (8) is nonconvex with respect to the variables $p_{1}$ and $p_{2}$. Nonetheless, we will show that it admits an optimal solution in a semi-closed form.

First, we present the following lemma, which helps to simplify the problem form.

Lemma 1 The optimal solution to problem (8) must activate the inequality constraint in Eq. (8b).

Proof Assume that an optimal solution $\left(p_{1}^{\prime}, p_{2}^{\prime}\right)$ satisfies $\sqrt{p_{1}^{\prime}}+\sqrt{p_{2}^{\prime}}<P_{o}$. It is evident that there always exists a positive number $\xi$ such that $\sqrt{p_{1}^{\prime}+\xi}+\sqrt{p_{2}^{\prime}}=P_{o}$ holds. Furthermore, as function $R_{1}\left(p_{1}, p_{2}\right)$ increases monotonically with $p_{1}$, $\left(p_{1}^{\prime}+\xi, p_{2}^{\prime}\right)$ fulfills the constraint in (8c); meanwhile, it achieves a larger objective value than $\left(p_{1}^{\prime}, p_{2}^{\prime}\right)$. This contradicts the optimality of $\left(p_{1}^{\prime}, p_{2}^{\prime}\right)$ and thus we complete the proof.

Let us denote $\sqrt{p_{2}}=\theta P_{o}$, where $0 \leqslant \theta \leqslant 1$. Then, we readily obtain $\sqrt{p_{1}}=(1-\theta) P_{o}$ from Lemma 1 . Therefore, problem (8) can be rewritten by

$$
\begin{aligned}
\max _{\theta} f(\theta)= & \frac{1}{2} \mathrm{lb}\left(1+\frac{2(1-\theta)^{2} h_{1}^{2} P_{o}^{2}}{\pi e\left(\theta^{2} h_{1}^{2} P_{o}^{2} / 3+\sigma_{z_{1}}^{2}\right)}\right) \\
& +\frac{1}{2} \operatorname{lb}\left(1+\frac{2 \theta^{2} h_{2}^{2} P_{o}^{2}}{\pi e \sigma_{z_{2}}^{2}}\right)
\end{aligned}
$$




$$
\begin{aligned}
& \text { s.t. } \\
& \left(\frac{\pi e\left(2^{2 \tilde{R_{1}}}-1\right)}{3}-2\right) \theta^{2}+4 \theta \\
& +\frac{\pi e\left(2^{2 \tilde{R_{1}}}-1\right) \sigma_{z_{1}}^{2}}{h_{1}^{2} P_{o}^{2}}-2 \leqslant 0, \\
& \frac{\pi e\left(2^{2 \tilde{R}_{2}}-1\right) \sigma_{z_{2}}^{2}}{2 h_{2}^{2} P_{o}^{2}}-\theta^{2} \leqslant 0, \\
& 0 \leqslant \theta \leqslant 1 \text {. }
\end{aligned}
$$

We analyze the feasibility condition of this problem in the following lemma.

Lemma 2 To guarantee the feasibility of the power allocation problem in (9), $\tilde{R}_{1}$ and $\tilde{R}_{2}$ should be selected such that

$$
\begin{aligned}
& \tilde{R}_{1} \leqslant \frac{1}{2} \mathrm{lb}\left(1+\frac{2 h_{1}^{2} P_{o}^{2}}{\pi e \sigma_{z_{1}}^{2}}\right), \\
& \tilde{R}_{2} \leqslant \frac{1}{2} \mathrm{lb}\left(1+\frac{2 \alpha^{2} h_{2}^{2} P_{o}^{2}}{\pi e \sigma_{z_{2}}^{2}}\right)
\end{aligned}
$$

hold, where $\alpha$ is given by

$$
\alpha= \begin{cases}\frac{-2+\sqrt{4-(X / 3-2)(X Y-2)}}{X / 3-2}, & \tilde{R}_{1} \neq \gamma, \\ \frac{1-3 Y}{2}, & \tilde{R}_{1}=\gamma,\end{cases}
$$

with

$$
\begin{aligned}
& X=\pi e\left(2^{2 \tilde{R}_{1}}-1\right), \quad Y=\frac{\sigma_{z_{1}}^{2}}{h_{1}^{2} P_{o}^{2}}, \\
& \gamma=\frac{1}{2} \operatorname{lb}\left(1+\frac{6}{\pi e}\right) .
\end{aligned}
$$

Proof With respect to (9b), let us denote its lefthand side by $g(\theta)$, and assume that $\tilde{R}_{1}>\gamma$. Then, it can be verified that

$$
\frac{\pi e}{3}\left(2^{2 \tilde{R_{1}}}-1\right)-2>0
$$

and

$$
g(1)=\pi e\left(2^{2 \tilde{R_{1}}}-1\right)\left(\frac{1}{3}+\frac{\sigma_{z_{1}}^{2}}{h_{1}^{2} P_{o}^{2}}\right)>0 .
$$

Furthermore, by considering that the axis of symmetry of the quadratic function $g(\theta)$ lies in the region $\theta<0$, we can infer that $g(0) \leqslant 0$ must hold because otherwise, (9b) will violate $(9 \mathrm{~d})$. Therefore, from $g(0) \leqslant 0$, we know that

$$
\tilde{R}_{1} \leqslant \frac{1}{2} \mathrm{lb}\left(1+\frac{2 h_{1}^{2} P_{o}^{2}}{\pi e \sigma_{z_{1}}^{2}}\right)
$$

must hold. Moreover, when $\tilde{R}_{1}<\gamma$, we obtain the same conclusion in an analogous manner, which is omitted here for brevity. For the case with $\tilde{R}_{1}=\gamma, g(\theta)$ becomes a linear function as $g(\theta)=4 \theta+6 \sigma_{z_{1}}^{2} /\left(h_{1}^{2} P_{o}^{2}\right)-2$. Clearly, to ensure that $(9 \mathrm{~b})$ does not violate $(9 \mathrm{~d})$, we also have $g(0) \leqslant 0$, which amounts to

$$
\tilde{R}_{1} \leqslant \frac{1}{2} \operatorname{lb}\left(1+\frac{2 h_{1}^{2} P_{o}^{2}}{\pi e \sigma_{z_{1}}^{2}}\right) .
$$

In order to fulfill the constraint (9b), we can deduce from the above analysis that $\theta$ cannot exceed the root of $g(\theta)=0$, which lies between 0 and 1 , and can be calculated by (10). On the other hand, by combining the constraint (9c) with $\theta \geqslant 0$, we have

$$
\theta \geqslant \sqrt{\frac{\pi e\left(2^{2 \tilde{R}_{2}}-1\right) \sigma_{z_{2}}^{2}}{2 h_{2}^{2} P_{o}^{2}}} \triangleq \beta .
$$

Therefore, we achieve another condition for guaranteeing the feasibility of problem (9) by $\beta \leqslant \alpha$ or equivalently,

$$
\tilde{R}_{2} \leqslant \frac{1}{2} \mathrm{lb}\left(1+\frac{2 \alpha^{2} h_{2}^{2} P_{o}^{2}}{\pi e \sigma_{z_{2}}^{2}}\right) .
$$

Theorem 1 The optimal solution to problem (9) can be expressed by

$$
\theta^{*}=\underset{\theta \in\{\mathbb{S} \cap[\beta, \alpha], \alpha, \beta\}}{\arg \max } f(\theta),
$$

where $\mathbb{S}$ denotes the set of all stationary points of the objective function $f(\theta)$. The stationary points of $f(\theta)$ are the roots of the following equation:

$$
\begin{aligned}
& \frac{\pi e}{3}\left(\frac{\pi e}{6}+1\right) \theta^{5}-\frac{\pi e}{3} \theta^{4} \\
& +\left(\frac{2 \pi e}{3}+4\right) A \theta^{3}+\left(\frac{\pi e}{3} B-6 A\right) \theta^{2} \\
& +\left[2 B\left(A-\frac{\pi e}{6}\right)+2 A(A+1)\right] \theta-2 A B=0,
\end{aligned}
$$

where

$$
A=\frac{\pi e \sigma_{z_{1}}^{2}}{2 h_{1}^{2} P_{o}^{2}}, \quad B=\frac{\pi e \sigma_{z_{2}}^{2}}{2 h_{2}^{2} P_{o}^{2}} .
$$

$\alpha$ and $\beta$ represent two boundary points of the feasible set whose expressions have been given in Eq. (10) and Eq. (11), respectively. 
Proof To determine the optimal solution to the scalar optimization problem in (9), we only need to concentrate on all feasible stationary points and all boundary points of the feasible set. According to the proof of Lemma 2 , the feasible set is $[\beta, \alpha]$. Furthermore, by letting $\mathrm{d} f(\theta) / \mathrm{d} \theta=0$ and performing a few tedious mathematical manipulations, we could show that all stationary points of the objective function $f(\theta)$ satisfy Eq. (12). Therefore, Theorem 1 is proved.

Finally, with $\theta^{*}$ available, we are able to calculate the optimal $p_{1}^{*}$ and $p_{2}^{*}$ as follows:

$$
\begin{gathered}
p_{1}^{*}=\left(1-\theta^{*}\right)^{2} P_{o}^{2}, \\
p_{2}^{*}=\left(\theta^{*}\right)^{2} P_{o}^{2} .
\end{gathered}
$$

\subsection{Max-min fairness power allocation}

As opposed to the previous subsection, here, we adopt an alternative max-min fairness criterion to optimize the power allocation. Specifically, we maximize the minimum user rate subject to the optical power and QoS constraints, whose mathematical form is

$$
\begin{array}{rc}
\max _{p_{1}, p_{2}} \min _{i=1,2} & R_{i} \\
\text { s.t. } & \sqrt{p_{1}}+\sqrt{p_{2}} \leqslant P_{o}, \\
& R_{1}\left(p_{1}, p_{2}\right) \geqslant \tilde{R}_{1}, \\
& R_{2}\left(p_{2}\right) \geqslant \tilde{R}_{2},
\end{array}
$$

where $R_{i}, i=1,2$ is given by (7). Although problem (15) is still nonconvex as problem (8), it also admits a semi-closed form solution, which is explained as follows.

First, by adopting the similar techniques of proving Lemma 1, we can show that the constraint (15b) is also active at optimality. Accordingly, by following the steps in the previous subsection and utilizing the increasing monotonicity of the logarithmic function, we transform problem (15) into an equivalent form as

$$
\max _{\theta} h(\theta)=\min \left\{\frac{2(1-\theta)^{2} h_{1}^{2} P_{o}^{2}}{\pi e\left(\theta^{2} h_{1}^{2} P_{o}^{2} / 3+\sigma_{z_{1}}^{2}\right)}, \frac{2 \theta^{2} h_{2}^{2} P_{o}^{2}}{\pi e \sigma_{z_{2}}^{2}}\right\}
$$

s.t.

$$
\begin{aligned}
& \left(\frac{\pi e\left(2^{2 \tilde{R_{1}}}-1\right)}{3}-2\right) \theta^{2}+4 \theta \\
& \quad+\frac{\pi e\left(2^{2 \tilde{R_{1}}}-1\right) \sigma_{z_{1}}^{2}}{h_{1}^{2} P_{o}^{2}}-2 \leqslant 0, \\
& \frac{\pi e\left(2^{2 \tilde{R_{2}}}-1\right) \sigma_{z_{2}}^{2}}{2 h_{2}^{2} P_{o}^{2}}-\theta^{2} \leqslant 0, \\
& 0 \leqslant \theta \leqslant 1 .
\end{aligned}
$$

Clearly, the feasibility condition in Lemma 2 also applies to the above problem. Furthermore, as a counterpart of Theorem 1, we show its optimal solution in the following theorem.

Theorem 2 The optimal solution to problem (16) is given by

$$
\theta^{*}=\underset{\theta \in\{\tilde{\theta} \cap[\beta, \alpha], \alpha, \beta\}}{\arg \max } h(\theta),
$$

where $\tilde{\theta} \in[0,1]$ is the unique root of the equation

$$
\frac{(1-\theta)^{2}}{\pi e \theta^{2} / 6+A}=\frac{\theta^{2}}{B} \text {. }
$$

Proof It has been shown in Lemma 2 that the feasible set is $[\beta, \alpha]$. Hence, the remaining work is to find the value of $\theta$ that maximizes the objective function $h(\theta)$. Before proceeding, we define

$$
h_{1}(\theta)=\frac{2(1-\theta)^{2} h_{1}^{2} P_{o}^{2}}{\pi e\left(\theta^{2} h_{1}^{2} P_{o}^{2} / 3+\sigma_{z_{1}}^{2}\right)}=\frac{(1-\theta)^{2}}{\pi e \theta^{2} / 6+A},
$$

and

$$
h_{2}(\theta)=\frac{2 \theta^{2} h_{2}^{2} P_{o}^{2}}{\pi e \sigma_{z_{2}}^{2}}=\frac{\theta^{2}}{B} .
$$

Then, it holds true that when $0 \leqslant \theta \leqslant 1, h_{1}(\theta)$ and $h_{2}(\theta)$ strictly decreases and increases with respect to $\theta$, respectively. Moreover, because $h_{1}(0)=1 / A>0$, $h_{1}(1)=0, h_{2}(0)=0$, and $h_{2}(1)=1 / B>0$, we readily have

$$
h(\theta)= \begin{cases}h_{2}(\theta), & \theta \leqslant \tilde{\theta} \\ h_{1}(\theta), & \theta>\tilde{\theta}\end{cases}
$$

where $\tilde{\theta} \in[0,1]$ is the unique root of $h_{1}(\theta)=h_{2}(\theta)$ and can be found by performing a bi-section search over the interval $[0,1]$. We can clearly see that $h(\theta)$ first increases and then decreases, and its maximum 
value is achieved at $\theta=\tilde{\theta}$. Thus far, we have proved Theorem 2.

Note that after obtaining $\theta^{*}$, the optimal $p_{1}^{*}$ and $p_{2}^{*}$ can also be achieved by Eq. (13) and Eq. (14), respectively.

\section{Simulation results}

In this section, we present the simulation results to demonstrate the performance of the proposed NOMA power allocation strategies. In order to show the superiority of NOMA, we consider the conventional OMA transmission as a benchmark, where the two users communicate with the LED in two timeslots. The detailed simulation parameters are listed in Tab. 1.

Table 1 Simulation parameters

\begin{tabular}{cc}
\hline parameters & values \\
\hline room size (length $\times$ width $\times$ height) & $5 \mathrm{~m} \times 5 \mathrm{~m} \times 3 \mathrm{~m}$ \\
number of LEDs per array & $(2.5 \mathrm{~m}, 2.5 \mathrm{~m}, 3 \mathrm{~m})$ \\
LED pitch & $3600(60 \times 60)$ \\
LED Lambertian emission order, $m$ & $1 \mathrm{~cm}$ \\
PD detection area, $A$ & 1 \\
PD responsivity, $R_{P}$ & $1 \mathrm{~cm}{ }^{2}$ \\
optical filter gain, $T$ & $0.4 \mathrm{~A} / \mathrm{W}$ \\
reflective index, $n$ & 1 \\
receiver FOV,$\Psi_{\mathrm{FOV}}$ & 1.5 \\
iamp & $62^{\circ}$ \\
pre-amplifier noise current density, & $10.93 \mathrm{~A} / \mathrm{m}^{2} \cdot \mathrm{Sr}^{-1}$ \\
ambient light photocurrent, & $5 \mathrm{pA} / \mathrm{Hz}^{1 / 2}$ \\
& $30 \mathrm{MHz}$ \\
\hline
\end{tabular}

Fig. 2 shows the achievable rate of each user and the sum rate for the NOMA scenario, where we set the rate threshold as $\tilde{R}_{1}=\tilde{R}_{2}=0.5 \mathrm{bit} / \mathrm{s} \cdot \mathrm{Hz}^{-1}$, and the coordinates of two users are $(1 \mathrm{~m}, 0 \mathrm{~m}, 0.85 \mathrm{~m})$ and $(2 \mathrm{~m}, 3 \mathrm{~m}, 0.85 \mathrm{~m})$, respectively. We can see from Fig. 2 that the respective rates of user 1 and user 2 decrease and increase with respect to the optimization variable $\theta$. On the other hand, the sum rate first increases, then decreases, and finally increases with $\theta$. When both users' rates are above the threshold value, i.e., the power allocation problem is feasible, the solution that maximizes the sum rate is a stationary point in the feasible set, while the max-min fairness is achieved when the rates of two users are equal.

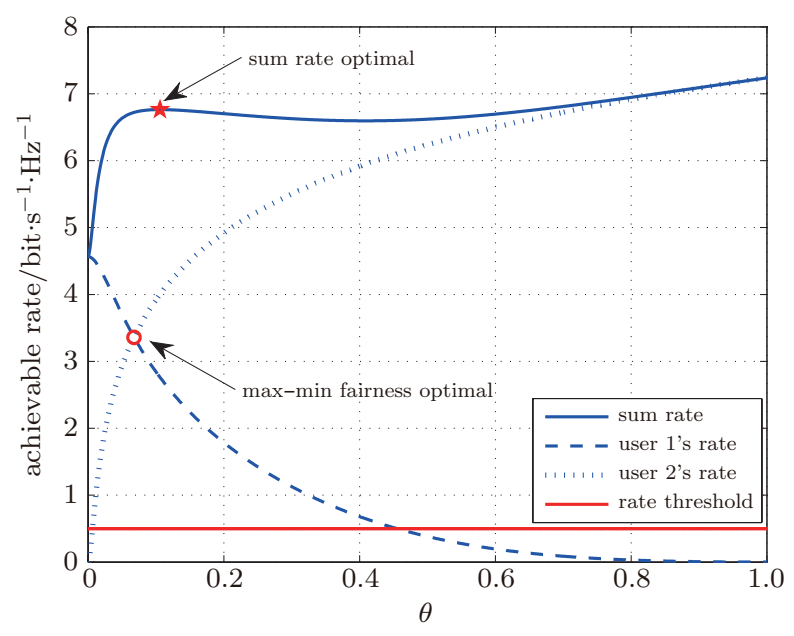

Figure 2 Individual and sum achievable rates versus optimization variable $\theta$

Fig. 3 compares the sum rates of NOMA and OMA schemes where $\tilde{R}_{1}=\tilde{R}_{2}=0.5 \mathrm{bit} / \mathrm{s} \cdot \mathrm{Hz}^{-1}$. For OMA, all available power is allocated to each user such that the sum rate is maximized. We consider two sets of user coordinates: 1) $(1 \mathrm{~m}, 0 \mathrm{~m}, 0.85 \mathrm{~m})$ and $(2 \mathrm{~m}, 3 \mathrm{~m}, 0.85 \mathrm{~m})$ (Coordinate I); 2$)(1 \mathrm{~m}, 4 \mathrm{~m}$, $0.85 \mathrm{~m})$ and $(2 \mathrm{~m}, 3 \mathrm{~m}, 0.85 \mathrm{~m})$ (Coordinate II). Compared to Coordinate I, the difference between the two users' channels corresponding to Coordinate II is small. It can be found that the sum rate of the NOMA strategy is clearly larger than that of the OMA scheme, especially when the optical power is relatively high. Moreover, the rate gain achieved by NOMA is more evident under Coordinate I, which means that NOMA is more advantageous when the user channels are very distinct. Note that we also apply the GP (Gradient Projection) algorithm (used in Ref. [12]) to solve problem (8), which can achieve almost the same sum rate as the proposed closedform solution in section 3.1. Nevertheless, the GP algorithm has a higher computational and implemen- 
tation complexity owing to the iterative mechanism and the fact that one convex problem needs to be solved in each iteration ${ }^{[12]}$.

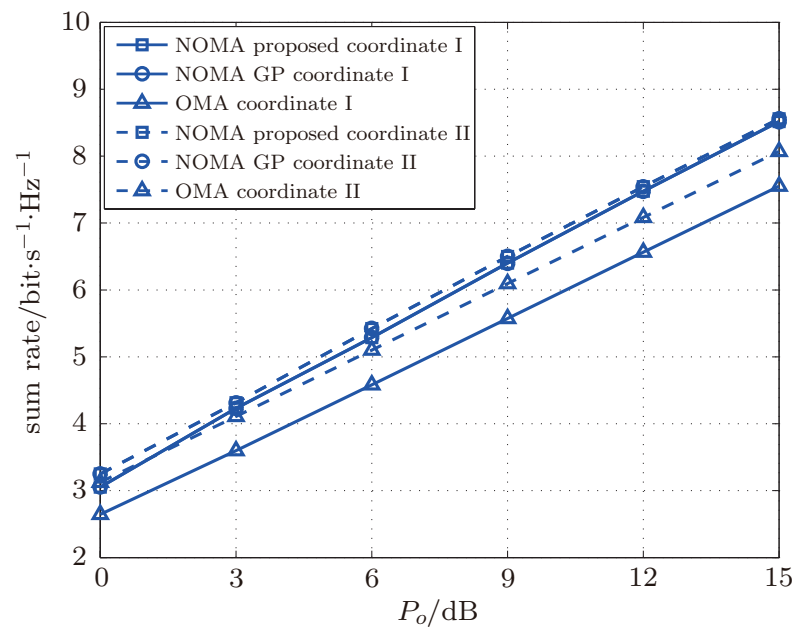

Figure 3 Performance comparison for NOMA and OMA schemes under sum-rate maximization criterion

We compared the max-min fairness rate for the NOMA and OMA schemes in Fig. 4, where we utilized the same parameters as in Fig. 3. The maxmin fairness based optimal power allocation in section 3.2 is used for NOMA. For the benchmark method OMA, we allocated the maximum available power to the user with a smaller signal-to-noise ratio, while ensuring that both users achieve identical rates. Similar to the results in Fig. 3, we observe in

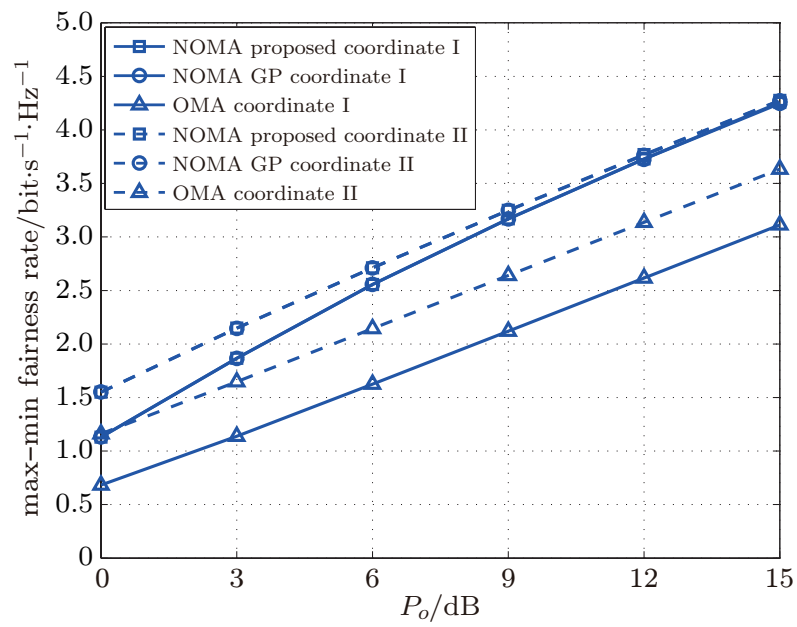

Figure 4 Performance comparison for NOMA and OMA schemes under max-min fairness criterion
Fig. 4 that NOMA still outperforms OMA in terms of max-min fairness. Moreover, the GP method and the proposed closed-form solution in section 3.2 achieve similar performances, while the GP algorithm has a higher complexity.

\section{Conclusions}

In this study, we investigated the optimal power allocation strategies for downlink NOMA VLC. We adopted both the sum-rate maximization and maxmin fairness criteria to conduct power allocation optimization for the two-user scenario. Despite the nonconvexity of the corresponding problems, we successfully determined their semi-closed form optimal solutions. We performed simulations, and the results verified the superiority of the proposed NOMA schemes for VLC downlinks. Future research directions include considering NLOS and channel estimation errors in order to improve the system performance in realistic scenarios. Moreover, we also hope to investigate the power allocation optimization using newer and more complicated rate bounds.

\section{References}

[1] L. Grobe, A. Paraskevopoulos, J. Hilt, et al. High-speed visible light communication systems [J]. IEEE communications magazine, 2013, 51(12): 60-66.

[2] D. Karunatilaka, F. Zafar, V. Kalavally, et al. LED based indoor visible light communications: state of the art [J]. IEEE communication surveys \& tutorials, 2015, 17(3): 1649-1678.

[3] H. Haas, L. Yin, Y. L. Wang, et al. What is LiFi? [J]. Journal of lightwave technology, 2016, 34(6): 1533-1544.

[4] Y. Saito, Y. Kishiyama, A. Benjebbour, et al. Nonorthogonal multiple access (NOMA) for cellular future radio access [C]//IEEE 77th Vehicular Technology Conference (VTC Spring), Dresden, c2013: 1-5.

[5] Y. Saito, A. Benjebbour, Y. Kishiyama, et al. System-level performance evaluation of downlink nonorthogonal multiple access (NOMA) [C]//IEEE 24th International Symposium on Personal Indoor and Mobile Radio Communications (PIMRC), London, c2013: 1-5.

[6] Z. G. Ding, Z. Yang, P. Z. Fan, et al. On the performance of non-orthogonal multiple access in $5 \mathrm{G}$ systems with randomly deployed users [J]. IEEE signal processing letters, 2014, 21(12): 1501-1505. 
[7] S. Timotheou, I. Krikidis. Fairness for non-orthogonal multiple access in $5 \mathrm{G}$ systems [J]. IEEE signal processing letters, 2015, 22(10): 1647-1651.

[8] C. L. Wang, J. Y. Chen, Y. J. Chen. Power allocation for a downlink non-orthogonal multiple access system [J]. IEEE wireless communications letters, 2016, 5(5): 532-535.

[9] Z. Yang, Z. G. Ding, P. Z. Fan, et al. A general power allocation scheme to guarantee quality of service in downlink and uplink NOMA systems [J]. IEEE transactions on wireless communications, 2016, 15(11): 7244-7257.

[10] H. Marshoud, V. M. Kapinas, G. K. Karagiannidis, et al. Non-orthogonal multiple access for visible light communications [J]. IEEE photonics technology letters, 2016, 28(1): 51-54.

[11] Z. H. Yang, W. Xu, Y. R. Li. Fair non-orthogonal multiple access for visible light communication downlinks [J]. IEEE wireless communications letters, 2016, 6(1): 66-69.

[12] X. K. Zhang, Q. Gao, C. Gong, et al. User grouping and power allocation for NOMA visible light communication multi-cell networks [J]. IEEE communications letters, 2016, 21(4): 777-780.

[13] J. M. Kahn, J. R. Barry. Wireless infrared communications [J]. Proceedings of the IEEE, 1997, 85(2): 265-298.

[14] L. Zeng, D. C. O'Brien, H. L. Minh, et al. High data rate multiple input multiple output (MIMO) optical wireless communications using white LED lighting [J]. IEEE journal on selected areas in communications, 2009, 27(9): 1654-1662.

[15] H. Shen, Y. Q. Deng, W. Xu, et al. Rate maximization for downlink multiuser visible light communications $[\mathrm{J}]$. IEEE access, 2016, 4: 6567-6573.

\section{About the authors}

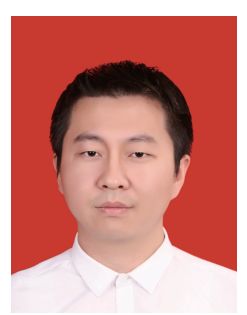

Hong Shen [corresponding author] was born in 1987. He received his B.E., M.S., and Ph.D. degrees in information and communication engineering from Southeast University, Nanjing, China, in 2009, 2011, and 2014, respectively. During his doctoral studies, he conducted cooperative research with the Department of Electrical and Computer Engineering, University of California, Davis, CA, USA. He is currently an associate professor with the National Mobile Communications Research Lab., Southeast University. His research interests include MIMO systems, optimization in wireless communications, visible light communications, and source localization in wireless sensor networks. (Email: shhseu@seu.edu.cn)

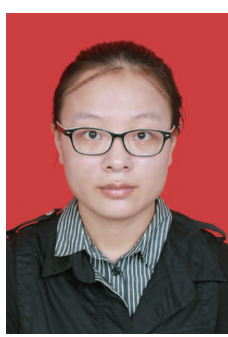

Yanfei Wu was born in 1994. She received her B.E. degree in information and communication engineering from Southeast University, Nanjing, China, in 2015, where she is currently working towards the M.S. degree in information and communication engineering. Her research interests include MIMO systems and visible light communications. (Email: yanfei_wu@seu.edu.cn)

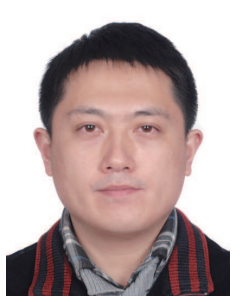

Wei Xu was born in 1982 . He received his B.Sc. degree in electrical engineering in 2003, and his M.S. and Ph.D. degrees in communication and information engineering in 2006 and 2009, respectively, all from Southeast University, Nanjing, China. He is currently a full professor at the National Mobile Communications Research Lab (NCRL), Southeast University. Between 2009 and 2010, he was a postdoctoral research fellow with the Department of Electrical and Computer Engineering, University of Victoria, Canada. Dr. Xu is an editor of the IEEE communications letters. He has been involved in technical program committees for many international conferences including IEEE Globecom, IEEE ICC, and IEEE WCNC. He has published over 100 refereed journal and conference papers in addition to 25 granted patents. He received the best paper awards of IEEE MAPE in 2013, IEEE/CIC ICCC in 2014, IEEE Globecom in 2014, and IEEE ICUWB in 2016. He was elected the core team member of the Jiangsu Innovation Team in 2012. He was the co-recipient of the First Prize Award of the Science and Technology Award in Jiangsu Province in 2014. His research interests include cooperative communications, information theory, and signal processing for wireless communications. (Email: wxu@seu.edu.cn)

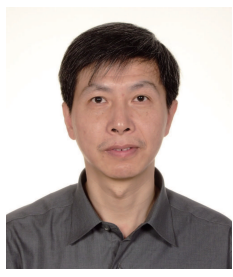

Chunming Zhao was born in 1959. He received his B.S. and M.S. degrees from Nanjing Institute of Posts and Telecommunications in 1982 and 1984, respectively. In 1993, he received his Ph.D. degree from the Department of Electrical and Electronic Engineering, University of Kaiserslautern, Germany. He has been a postdoctoral researcher at National Mobile Communications Research Lab., Southeast University, where he is currently a professor and the vice director of the Lab. He has managed several key projects of Chinese Communications High Tech. Program. His research interests include communication theory, coding/decoding, mobile communications, and VLSI design. Dr. Zhao won the First Prize of National Technique Invention of China in 2011. He was awarded "Excellent Researcher" from the Ministry of Science and Technology, China. (Email: cmzhao@seu.edu.cn) 